\title{
Generalized Mittag-Leffler Type Function: Fractional Integrations and Application to Fractional Kinetic Equations
}

\section{Kottakkaran Sooppy Nisar*}

Department of Mathematics, College of Arts and Sciences, Prince Sattam Bin Abdulaziz University, Wadi Aldawaser, Saudi Arabia

The generalized fractional integrations of the generalized Mittag-Leffler type function (GMLTF) are established in this paper. The results derived in this paper generalize many results available in the literature and are capable of generating several applications in the theory of special functions. The solutions of a generalized fractional kinetic equation using the Sumudu transform is also derived and studied as an application of the GMLTF.

Keywords: The Mittag-Leffler type function, fractional calculus, fractional kinetic equations; MSC [2000]: 33C05; 33C20; 33E12; 26A33; 44A20; 35Qxx, riemam-liouville derivative, special function

\section{OPEN ACCESS}

Edited by: Devendra Kumar University of Rajasthan, India

Reviewed by: Haci Mehmet Baskonus, Harran University, Turkey Yudhveer Singh, Amity University Jaipur, India

*Correspondence: Kottakkaran Sooppy Nisar n.sooppy@psau.edu.sa

Specialty section: This article was submitted to Mathematical Physics,

a section of the journal

Frontiers in Physics

Received: 21 December 2019 Accepted: 05 February 2020 Published: 28 February 2020

Citation:

Nisar KS (2020) Generalized Mittag-Leffler Type Function: Fractional Integrations and Application to Fractional Kinetic Equations. Front. Phys. 8:33. doi: 10.3389/fphy.2020.00033

\section{INTRODUCTION}

The Pochhammer symbol $(\varpi)_{n}$ is defined by (for $\left.\varpi \in \mathbb{C}\right)[$ see ([1], p. 2 and p. 5)]:

$$
\begin{aligned}
(\varpi)_{n}: & =\left\{\begin{array}{lr}
1 & (n=0) \\
\varpi(\varpi+1) \ldots(\varpi+n-1) & (n \in \mathbb{N})
\end{array}\right. \\
& =\frac{\Gamma(\varpi+n)}{\Gamma(\varpi)} \quad\left(\varpi \in \mathbb{C} \backslash \mathbb{Z}_{0}^{-}\right) .
\end{aligned}
$$

The familiar generalized hypergeometric function ${ }_{p} F_{q}$ is defined as follows (see [2]):

$$
\begin{gathered}
{ }_{p} F_{q}\left[\begin{array}{c}
\left(\varpi_{p}\right) ; \\
\left(\chi_{q}\right) ; x
\end{array}\right]=\sum_{n=0}^{\infty} \frac{\Pi_{j=1}^{p}\left(\varpi_{j}\right)_{n}}{\Pi_{j=1}^{q}\left(x_{j}\right)_{n}} \frac{x^{n}}{n !}, \\
(p \leq q, x \in \mathbb{C} ; p=q+1,|x|<1),
\end{gathered}
$$

where $\left(\varpi_{j}\right)_{n}$ and $\left(\chi_{j}\right)_{n}$ given in (1) and $\chi_{i}$ can not be a negative integer or zero. Here $p$ or $q$ or both are permitted to be zero. For all finite $x$, the series (2) is absolutely convergent if $p \leq q$ and for $|x|<1$ if $p=q+1$. When $p>q+1$, then the series diverge for $x \neq 0$ and the series does not terminate.

In particular, if $p=2$ and $q=1$, (2) reduces to the Gaussian hypergeometric function

$$
{ }_{2} F_{1}\left(\varpi_{1}, \varpi_{2} ; \varpi_{3} ; x\right)=\sum_{k=0}^{\infty} \frac{\left(\varpi_{1}\right)_{n}\left(\varpi_{2}\right)_{n}}{\left(\varpi_{3}\right)_{n}} \frac{x^{n}}{n !} .
$$


The function ${ }_{r} \Psi_{s}(z)$ is the generalized Wright hypergeometric series which is given by

$$
{ }_{r} \Psi_{s}(z)={ }_{r} \Psi_{s}\left[\begin{array}{c}
\left(\mathfrak{a}_{i}, \varpi_{i}\right)_{1, r} \\
\left(\mathfrak{b}_{j}, \chi_{j}\right)_{1, s}
\end{array} \mid z\right]=\sum_{k=0}^{\infty} \frac{\prod_{i=1}^{r} \Gamma\left(\mathfrak{a}_{i}+\varpi_{i} k\right)}{\prod_{j=1}^{s} \Gamma\left(\mathfrak{b}_{j}+\chi_{j} k\right)} \frac{z^{k}}{k !},
$$

where $\mathfrak{a}_{i}, \mathfrak{b}_{j} \in \mathbb{C}$, and real $\varpi_{i}, \chi_{j} \in \mathbb{R}(i=1,2, \ldots, r ; j=$ $1,2, \ldots, s)$. The asymptotic behavior of (4) for large values of argument of $z \in \mathbb{C}$ were mentioned in $[3,4]$ (also, see $[5,6]$ ).

To proceed our study, we need the definitions of the MittagLeffler functions (MLF) denoted by $E_{\varpi}(z)$ (see [7]) and $E_{\varpi, \chi}(x)$ [8], respectively:

$$
\begin{gathered}
E_{\varpi}(x)=\sum_{n=0}^{\infty} \frac{x^{n}}{\Gamma(\varpi n+1)} \quad(x, \varpi \in \mathbb{C} ;|x|<0, \Re(\varpi)>0) \\
E_{\varpi, \chi}(x)=\sum_{\substack{n=0 \\
\Re(\varpi)>0, \Re(\chi)>0) .}}^{\infty} \frac{x^{n}}{\Gamma(\varpi n+\chi)} \quad(x, \varpi, \chi \in \mathbb{C} ;
\end{gathered}
$$

Many more generalizations and extensions of MLF widely studied recently $[9,10]$. Also, the MLF performs an important role in physics and engineering problems. The derivations of physical problems of exponential nature could be governed by the physical laws through the MLF (power-law) [11-13].

Very recently, Nisar [14] defined a generalized Mittag-Leffler type function which is defined as follows

For $\rho, \sigma, \varsigma \in \mathbb{C}, \Re(\kappa)>0, \delta \neq 0,-1,-2, \cdots,(\kappa)_{s}$ and $(\omega)_{s}$ denotes the Pochammer symbol.

$$
\begin{aligned}
{ }_{p} \mathrm{E}_{q ; \delta}^{\rho, \sigma ; \varsigma}(z) & ={ }_{p} \mathrm{E}_{q, \delta}^{\rho, \sigma, \varsigma}\left(\kappa_{1}, \kappa_{2}, \cdots, \kappa_{p} ; \omega_{1}, \omega_{2}, \cdots, \omega_{q} ; z\right) \\
& =\sum_{s=0}^{\infty} \frac{\left(\kappa_{1}\right)_{s}\left(\kappa_{2}\right)_{s} \cdots\left(\kappa_{p}\right)_{s}}{\left(\omega_{1}\right)_{s}\left(\omega_{2}\right)_{s} \cdots\left(\omega_{q}\right)_{s}} \frac{(\varsigma)_{s} z^{s}}{(\delta)_{s} \Gamma(\rho s+\sigma)} .
\end{aligned}
$$

By assuming particular values for various parameters in (7), we get many of the popular functions in the literature. For example, ${ }_{p} \mathrm{E}_{q ; 1}^{\rho, \sigma ; \zeta}(z)$ gives the $K$ - function [15] and ${ }_{0} \mathrm{E}_{0 ; 1}^{\rho, \sigma ; \zeta}(z)$ turns to $E_{\rho, \sigma}^{\varsigma}(z)$ [16]. Also, ${ }_{0} \mathrm{E}_{0 ; \delta}^{\rho, \sigma ; \zeta}(z)$ reduces to $E_{\rho, \sigma}^{\zeta, \delta}(z)$ [17] and ${ }_{0} \mathrm{E}_{0 ; 1}^{\rho, \sigma ; 1}(z)$ gives the Mittag-Leffler function $E_{\rho, \sigma}(z)$ [8]. Similar way, ${ }_{0} \mathrm{E}_{0 ; 1}^{\rho, 1 ; 1}(z)$ turns to the Mittag-Leffler functions $E_{\rho}(z)$ [7]. For more details one can be referred to Nisar [14].

\section{GENERALIZED FRACTIONAL INTEGRATION OF GMLTF}

Fractional calculus is one of the prominent branch of applied mathematics that deals with non-integer order derivatives and integrals (including complex orders), and their applications in almost all disciplines of science and engineering [18-22]. In this line, the use of special functions in connection with fractional calculus also studied widely [23-27]. For the basics of fractional calculus and its related literature, interesting readers can be referred to as Kiryakova [28], Miller and Ross [29], and Srivastava et al. [30]. In this paper, we studied the generalized fractional calculus of more generalized function given in (7). The generalized fractional integral operators (FIOs) involving the Appell functions $F_{3}$ are given for $\varpi, \varpi^{\prime}, \tau, \tau^{\prime}, \epsilon \in \mathbb{C}$ with $\mathfrak{R}(\epsilon)>0$ and $x \in \mathbb{R}^{+}$as follows:

$$
\begin{aligned}
& \left(I_{0+}^{\varpi, \varpi^{\prime}, \tau, \tau^{\prime}, \epsilon} f\right)(x)=\frac{x^{-\varpi}}{\Gamma(\epsilon)} \int_{0}^{x}(x-t)^{\epsilon-1} t^{-\varpi^{\prime}} \\
& F_{3}\left(\varpi, \varpi^{\prime}, \tau, \tau^{\prime} ; \epsilon ; 1-\frac{t}{x}, 1-\frac{x}{t}\right) f(t) \mathrm{d} t
\end{aligned}
$$

and

$$
\begin{aligned}
& \left(I_{-}^{\varpi, \varpi^{\prime}, \tau, \tau^{\prime}, \epsilon} f\right)(x)=\frac{x^{-\varpi^{\prime}}}{\Gamma(\epsilon)} \int_{x}^{\infty}(t-x)^{\epsilon-1} t^{-\varpi} \\
& F_{3}\left(\varpi, \varpi^{\prime}, \tau, \tau^{\prime} ; \epsilon ; 1-\frac{t}{x}, 1-\frac{x}{t}\right) f(t) \mathrm{d} t
\end{aligned}
$$

The integral operators of the types (8) and (9) have been introduced by Marichev [31] and later extended and studied by Saigo and Maeda [32]. Recently, many researchers (see [33-35]) have studied the image formulas for MSM FIOs involving various special functions.

The corresponding fractional differential operators (FDOs) have their respective forms:

$$
\begin{aligned}
& \left(D_{0+}^{\varpi, \varpi^{\prime}, \tau, \tau^{\prime}, \epsilon} f\right)(x)=\left(\frac{\mathrm{d}}{\mathrm{d} x}\right)^{[\Re(\epsilon)]+1} \\
& \quad\left(I_{0+}^{-\varpi^{\prime},-\varpi,-\tau^{\prime}+[\Re(\epsilon)]+1,-\tau,-\epsilon+[\Re(\epsilon)]+1} f\right)(x)
\end{aligned}
$$

and

$$
\begin{aligned}
& \left(D_{-}^{\varpi, \varpi^{\prime}, \tau, \tau^{\prime}, \epsilon} f\right)(x)=\left(-\frac{\mathrm{d}}{\mathrm{d} x}\right)^{[\Re(\epsilon)]+1} \\
& \quad\left(I_{-}^{-\varpi^{\prime},-\varpi,-\tau^{\prime},-\tau+[\Re(\epsilon)]+1,-\epsilon+[\Re(\epsilon)]+1} f\right)(x) .
\end{aligned}
$$

Here, we recall the following results (see $[32,36]$ ).

LEMMA 2.1. Let $\varpi, \varpi^{\prime}, \tau, \tau^{\prime}, \epsilon, \sigma \quad \in \quad \mathbb{C}$ be such that $\Re(\epsilon)>0$ and

$$
\mathfrak{R}(\sigma)>\max \left\{0, \mathfrak{R}\left(\varpi+\varpi^{\prime}+\tau-\epsilon\right), \mathfrak{R}\left(\varpi^{\prime}-\tau^{\prime}\right)\right\} .
$$

Then there exists the relation

$$
\begin{aligned}
& \left(I_{0+}^{\varpi, \varpi^{\prime}, \tau, \tau^{\prime}, \epsilon} t^{\sigma-1}\right)(x) \\
& =\frac{\Gamma(\sigma) \Gamma\left(\sigma+\epsilon-\varpi-\varpi^{\prime}-\tau\right)}{\Gamma\left(\sigma+\tau^{\prime}-\varpi^{\prime}\right)} \\
& =\frac{\Gamma\left(\sigma+\tau^{\prime}\right) \Gamma\left(\sigma+\epsilon-\varpi-\varpi^{\prime}\right)}{\Gamma\left(\sigma+\epsilon-\varpi^{\prime}-\tau\right)} x^{\sigma-\varpi-\varpi^{\prime}+\epsilon-1} .
\end{aligned}
$$

LEMMA 2.2. Let $\varpi, \varpi^{\prime}, \tau, \tau^{\prime}, \epsilon, \sigma \in \mathbb{C}$ such that $\Re(\epsilon)>0$ and

$$
\Re(\sigma)>\max \left\{\Re(\tau), \mathfrak{R}\left(-\varpi-\varpi^{\prime}+\epsilon\right), \mathfrak{R}\left(-\varpi-\tau^{\prime}+\epsilon\right)\right\} .
$$


Then

$$
\begin{aligned}
& \left(I_{-}^{\varpi, \varpi^{\prime}, \tau, \tau^{\prime}, \epsilon} t^{-\sigma}\right)(x) \\
& =\frac{\Gamma(-\tau+\sigma) \Gamma\left(\varpi+\varpi^{\prime}-\epsilon+\sigma\right)}{\Gamma\left(\varpi+\tau^{\prime}-\epsilon+\sigma\right)} \\
& =\frac{\Gamma(\sigma) \Gamma(\varpi-\tau+\sigma)}{\Gamma\left(\varpi+\varpi^{\prime}+\tau^{\prime}-\epsilon+\sigma\right)} x^{-\varpi-\varpi^{\prime}+\epsilon-\sigma},
\end{aligned}
$$

The main aim of this paper is to apply the generalized operators of fractional calculus for the GMLTF in order to get certain new image formulas.

\subsection{Sumudu Transform}

The Sumudu transform is widely used to solve various type of problems in science and engineering and it is introduced by Watugala (see $[37,38]$ ). The details of Sumudu transforms, properties, and its applications the interesting readers can be refer to Asiru [39], Belgacem et al. [40], and Bulut et al. [41].

The Sumudu transform over the set functions

$A=\left\{f(t)\left|\exists M, \tau_{1}, \tau_{2}>0,\right| f(t) \mid<M e^{|t| / \tau_{j}}\right.$, if $\left.t \in(-1)^{j} \times[0, \infty)\right\}$,

is defined by

$$
G(u)=S[f(t) ; u]=\int_{0}^{\infty} f(u t) e^{-t} d t, u \in\left(-\tau_{1}, \tau_{2}\right) .
$$

The main aim of this study is to establish the generalized fractional calculus operators and the generalized FKEs involving GMLTF.

Theorem 2.1. Let $\eta, \eta^{\prime}, \chi, \chi^{\prime}, \epsilon, \tau, \varpi, \lambda, \gamma \in \mathbb{C}, \Re(\kappa)>0, \delta \neq$ $0,-1,-2, \cdots$, such that $\Re(\tau)>\max \left\{0, \Re\left(\eta+\eta^{\prime}-\chi-\epsilon\right), \mathfrak{R}\left(\eta^{\prime}-\right.\right.$ $\left.\left.\chi^{\prime}\right)\right\}$. Then

$$
\begin{aligned}
& \left(I_{0_{+}}^{\eta, \eta^{\prime}, \chi, \chi^{\prime}, \epsilon} t^{\tau-1}{ }_{p} E_{q ; \delta}^{\varpi, \lambda ; \gamma}(t)\right)(x) \\
& =\frac{\Gamma(\delta) \prod_{j=1}^{q} \Gamma\left(\omega_{j}\right)}{\Gamma(\gamma) \prod_{i=1}^{p} \Gamma\left(\kappa_{i}\right)} x^{\tau-\eta-\eta^{\prime}+\epsilon-1} \times_{p+5} \Psi_{q+5} \\
& {\left[\begin{array}{c}
\left(\kappa_{i}, 1\right)_{1, p},(\gamma, 1),(\tau, 1),\left(\tau+\epsilon-\eta-\eta^{\prime}-\chi, 1\right), \\
\left(\tau+\chi^{\prime}-\eta^{\prime}, 1\right),(1,1) \\
\left(\omega_{j}, 1\right)_{1, q},(\delta, 1),(\gamma, \varpi),\left(\tau+\chi^{\prime}, 1\right),\left(\tau+\epsilon-\eta-\eta^{\prime}, 1\right), \\
\left(\tau+\epsilon-\eta^{\prime}-\chi, 1\right)
\end{array}\right] .}
\end{aligned}
$$

Proof: Applying the definition (7) on the left hand side (l.h.s) of Theorem 2.1,

$$
\begin{aligned}
& \mathfrak{I}_{1}=\left(I_{0_{+}}^{\eta, \eta^{\prime}, \chi, \chi^{\prime}, \epsilon} t^{\tau-1}{ }_{p} E_{q ; \delta}^{\varpi, \lambda ; \gamma}(t)\right)(x) \\
& =\left(I_{0_{+}}^{\eta, \eta^{\prime}, \chi, \chi^{\prime}, \epsilon} t^{\tau-1} \sum_{r=0}^{\infty} \frac{\left(\kappa_{1}\right)_{r}\left(\kappa_{2}\right)_{r} \cdots\left(\kappa_{p}\right)_{r}}{\left(\omega_{1}\right)_{r}\left(\omega_{2}\right)_{r} \cdots,\left(\omega_{q}\right)_{r}} \frac{(\gamma)_{r} t^{r}}{(\delta)_{r} \Gamma(\varpi r+\lambda)}\right)(x)
\end{aligned}
$$

Changing the order of integration and summation gives

$\mathfrak{I}_{\perp}=\sum_{r=0}^{\infty} \frac{\left(\kappa_{1}\right)_{r}\left(\kappa_{2}\right)_{r} \cdots\left(\kappa_{p}\right)_{r}}{\left(\omega_{1}\right)_{r}\left(\omega_{2}\right)_{r} \cdots\left(\omega_{q}\right)_{r}} \frac{(\gamma)_{r}}{(\delta)_{r} \Gamma(\varpi r+\lambda)}\left(I_{0_{+}}^{\eta, \eta^{\prime}, x, \chi^{\prime}, \epsilon} t^{\tau+r-1}\right)(x)$

Applying Lemma 2.1, we get

$$
\begin{gathered}
\mathfrak{I}_{1}=\sum_{r=0}^{\infty} \frac{\left(\kappa_{1}\right)_{r}\left(\kappa_{2}\right)_{r} \cdots\left(\kappa_{p}\right)_{r}}{\left(\omega_{1}\right)_{r}\left(\omega_{2}\right)_{r} \cdots\left(\omega_{q}\right)_{r}} \frac{(\gamma)_{r}}{(\delta)_{r} \Gamma(\varpi r+\lambda)} \\
\times \frac{\Gamma(\tau+r)}{\left(\tau+r+\epsilon-\eta-\eta^{\prime}-\chi\right) \Gamma\left(\tau+r+\chi^{\prime}-\eta^{\prime}\right)} \\
\begin{array}{c}
\left(\Gamma+r+\chi^{\prime}\right) \Gamma\left(\tau+r+\epsilon-\eta-\eta^{\prime}\right) \\
\Gamma\left(\tau+r+\epsilon-\eta^{\prime}-\chi\right)
\end{array}
\end{gathered}
$$

Using $\Gamma(x+\kappa)=(x)_{k} \Gamma(x)$, we have

$$
\begin{gathered}
\mathfrak{I}_{1}=x^{\tau-\eta-\eta^{\prime}+\epsilon-1} \frac{\Gamma(\delta) \prod_{j=1}^{q} \Gamma\left(\omega_{j}\right)}{\Gamma(\gamma) \prod_{i=1}^{p} \Gamma\left(\kappa_{i}\right)} \sum_{r=0}^{\infty} \frac{\prod_{i=1}^{p} \Gamma\left(\kappa_{i}+r\right)}{\prod_{j=1}^{q} \Gamma\left(\omega_{j}+r\right)} \\
\times x^{r} \frac{\Gamma(\gamma+r) \Gamma(\tau+r) \Gamma\left(\tau+r+\epsilon-\eta-\eta^{\prime}-\chi\right)}{\Gamma(1+r) \Gamma(\delta+r) \Gamma(\varpi r+\lambda) \Gamma\left(\tau+\chi^{\prime}+r\right)} \\
\Gamma\left(\tau+\epsilon+r-\eta-\eta^{\prime}\right) \\
\Gamma\left(\tau+\epsilon+r-\eta^{\prime}-\chi\right)
\end{gathered}
$$

In view of (4), we reached the required result.

Theorem 2.2. Let $\eta, \eta^{\prime}, \chi, \chi^{\prime}, \epsilon, \tau, \varpi, \lambda, \gamma \in \mathbb{C}, \Re(\kappa)>0, \delta \neq$ $0,-1,-2, \cdots$, such that $\mathfrak{R}(\tau)>\max \left\{\mathfrak{R}(\chi), \mathfrak{R}\left(-\eta-\eta^{\prime}+\right.\right.$ $\left.\epsilon), \Re\left(-\eta-\chi^{\prime}+\epsilon\right)\right\}$. Then

$$
\begin{aligned}
& \left(I_{-}^{\eta, \eta^{\prime}, \chi, \chi^{\prime}, \epsilon} t_{p}^{-\tau} E_{q ; \delta} E^{\varpi, \lambda ; \gamma}\left(\frac{1}{t}\right)\right)(x) \\
& =\frac{\Gamma(\delta) \prod_{j=1}^{q} \Gamma\left(\omega_{j}\right)}{\Gamma(\gamma) \prod_{i=1}^{p} \Gamma\left(\kappa_{i}\right)} x^{-\eta-\eta^{\prime}+\epsilon-\tau} \\
& \times_{p+5} \Psi_{q+5}\left[\begin{array}{c}
\left(\kappa_{i}, 1\right)_{1, p},(\gamma, 1),(-\chi+\tau, 1), \\
\left(\eta+\eta^{\prime}-\epsilon+\tau, 1\right),\left(\eta+\chi^{\prime}-\epsilon+\tau, 1\right),(1,1) \\
\left(\omega_{j}, 1\right)_{1, q},(\delta, 1),(\lambda, \varpi),(\tau, 1), \\
(\eta-\chi+\tau, 1),\left(\eta+\eta^{\prime}+\chi^{\prime}-\epsilon+\tau, 1\right)
\end{array} \mid x\right] .
\end{aligned}
$$

Proof: Applying the definition (7) on the left hand side (l.h.s) of Theorem 2.2,

$$
\begin{aligned}
& \mathfrak{I}_{2}=\left(I_{-}^{\eta, \eta^{\prime}, \chi, \chi^{\prime}, \epsilon} t_{p}^{-\tau} E_{q ; \delta}^{\varpi, \lambda ; \gamma}\left(\frac{1}{t}\right)\right)(x) \\
& =\left(I_{-}^{\eta, \eta^{\prime}, \chi, \chi^{\prime}, \epsilon} t^{-\tau} \sum_{r=0}^{\infty} \frac{\left(\kappa_{1}\right)_{r}\left(\kappa_{2}\right)_{r} \cdots\left(\kappa_{p}\right)_{r}}{\left(\omega_{1}\right)_{r}\left(\omega_{2}\right)_{r} \cdots\left(\omega_{q}\right)_{r}} \frac{(\gamma)_{r} t^{r}}{(\delta)_{r} \Gamma(\varpi r+\lambda)}\right)(x)
\end{aligned}
$$

Changing the order of integration and summation gives

$\mathfrak{I}_{2}=\sum_{r=0}^{\infty} \frac{\left(\kappa_{1}\right)_{r}\left(\kappa_{2}\right)_{r} \cdots\left(\kappa_{p}\right)_{r}}{\left(\omega_{1}\right)_{r}\left(\omega_{2}\right)_{r} \cdots\left(\omega_{q}\right)_{r}} \frac{(\gamma)_{r}}{(\delta)_{r} \Gamma(\varpi r+\lambda)}\left(I_{-}^{\eta, \eta^{\prime}, \chi, \chi^{\prime}, \epsilon} t^{-\tau-r}\right)(x)$ 
Applying Lemma 2.2, we get

$$
\begin{gathered}
\mathfrak{I}_{2}=\sum_{r=0}^{\infty} \frac{\left(\kappa_{1}\right)_{r}\left(\kappa_{2}\right)_{r} \cdots\left(\kappa_{p}\right)_{r}}{\left(\omega_{1}\right)_{r}\left(\omega_{2}\right)_{r} \cdots\left(\omega_{q}\right)_{r}} \frac{(\gamma)_{r}}{(\delta)_{r} \Gamma(\varpi r+\lambda)} \\
\times \frac{\Gamma(-\chi+\tau+r) \Gamma\left(\eta+\eta^{\prime}-\epsilon+\tau+r\right)}{\Gamma\left(\eta+\chi^{\prime}-\epsilon+\tau+r\right)} \\
\Gamma(\tau+r) \Gamma(\eta-\chi+\tau+r) \\
\Gamma\left(\eta+\eta^{\prime}+\chi^{\prime}-\epsilon+\tau+r\right)
\end{gathered} .
$$

Using $\Gamma(x+r)=(x)_{r} \Gamma(x)$, we have

$$
\begin{gathered}
\mathfrak{I}_{2}=x^{-\eta-\eta^{\prime}+\epsilon-\tau} \frac{\Gamma(\delta) \prod_{j=1}^{q} \Gamma\left(\omega_{j}\right)}{\Gamma(\gamma) \prod_{i=1}^{p} \Gamma\left(\kappa_{i}\right)} \sum_{r=0}^{\infty} \frac{\prod_{i=1}^{p} \Gamma\left(\kappa_{i}+r\right)}{\prod_{j=1}^{q} \Gamma\left(\omega_{j}+r\right)} \\
\times x^{r} \frac{\Gamma(\gamma+r) \Gamma(-\chi+\tau+r) \Gamma\left(\eta+\eta^{\prime}-\epsilon+\tau+r\right)}{\Gamma(\delta+r) \Gamma(\varpi r+\lambda) \Gamma(\tau+r) \Gamma(\eta-\chi+\tau+r)} \\
\Gamma\left(\eta+\eta^{\prime}+\chi^{\prime}-\epsilon+\tau+r\right)
\end{gathered}
$$

In view of (4), we reached the required result.

The following corollaries can derive immediately from Theorems 2.1 and 2.2 with the help of Pochhammer symbol

Corollary 2.1. Let $\delta=\lambda=1$ in Theorem 2.1, we get

$$
\begin{aligned}
& \left(I_{0_{+}}^{\eta, \eta^{\prime}, \chi, \chi^{\prime}, \epsilon} t^{\tau-1}{ }_{p} \mathrm{E}_{q ; \delta}^{\varpi, \lambda ; \gamma}(t)\right)(x) \\
= & \frac{\Gamma(\tau) \Gamma\left(\tau+\epsilon-\eta-\eta^{\prime}-\chi\right) \Gamma\left(\tau+\chi^{\prime}-\eta^{\prime}\right)}{\Gamma\left(\tau+\chi^{\prime}\right) \Gamma\left(\tau+\epsilon-\eta-\eta^{\prime}\right)} x^{\tau-\eta-\eta^{\prime}+\epsilon-1} \\
\Gamma\left(\tau+\epsilon-\eta^{\prime}-\chi\right) \Gamma(\gamma) & {\left[\begin{array}{c}
\left(\kappa_{i}, 1\right)_{1, p}, \nu, \tau, \tau+\epsilon-\eta-\eta^{\prime}-\chi, \\
\tau+\chi^{\prime}-\eta^{\prime} ; \\
\left(\omega_{j}, 1\right)_{1, q}, \lambda, \tau+\chi^{\prime}, \tau+\epsilon-\eta-\eta^{\prime}, \\
\tau+\epsilon-\eta^{\prime}-\chi ;
\end{array}\right] . }
\end{aligned}
$$

Corollary 2.2. If $\delta=\lambda=1$ in Theorem 2.2, then

$$
\begin{aligned}
& \left(I_{-}^{\eta, \eta^{\prime}, \chi, \chi^{\prime}, \epsilon} t^{\tau-1}{ }_{p} \mathrm{E}_{q ; \delta}^{\varpi, \lambda ; \gamma}\left(\frac{1}{t}\right)\right)(x) \\
& \Gamma(\tau-\chi) \Gamma\left(\eta+\eta^{\prime}-\epsilon+\tau\right) \\
& =\frac{\Gamma\left(\eta+\chi^{\prime}-\epsilon+\tau\right)}{\Gamma(\tau) \Gamma(\eta-\chi+\tau) \Gamma\left(\eta+\eta^{\prime}+\chi-\epsilon+\tau\right)} x^{-\tau-\eta-\eta^{\prime}+\epsilon-1} \\
& \Gamma(\gamma) \\
& \times_{p+4} F_{q+4}\left[\begin{array}{c|c}
\left(\kappa_{i}, 1\right)_{1, p}, \gamma, \tau-\chi, \eta+\eta^{\prime}-\epsilon+\tau, \eta+\chi^{\prime} \\
-\epsilon+\tau ; \\
\left(\omega_{j}, 1\right)_{1, q}, \lambda, \tau, \eta-\chi+\tau, \eta+\eta^{\prime} \\
-\epsilon+\tau ;
\end{array} \mid x\right] .
\end{aligned}
$$

In the next section, we derived the generalized FKEs and we consider the Sumudu transform methodology to achieve the results.

\section{GENERALIZED FRACTIONAL KINETIC EQUATIONS INVOLVING GMLTF}

The generalized fractional kinetic equations (FKEs) involving the GMLTF with the Sumudu transform is derived in this section. The FKEs are studied widely in many papers [42-45].

Let $\mathfrak{K}=\left(\mathfrak{K}_{t}\right)$ be the arbitrary reaction defined by a timedependent quantity. The destruction $\mathfrak{d}$ and production $\mathfrak{p}$ depend on the quantity $\mathfrak{K}$ itself: $\mathfrak{d}=\mathfrak{d}(\mathfrak{K})$ or $\mathfrak{p}=\mathfrak{p}(\mathfrak{K})$ [see [42]]. The fractional differential equation can be expressed by

$$
\frac{d \mathfrak{K}}{d t}=-\mathfrak{d}\left(\mathfrak{K}_{t}\right)+\mathfrak{p}\left(\mathfrak{K}_{t}\right),
$$

where $\mathfrak{K}_{t}$ described by $\mathfrak{K}_{t}\left(t^{*}\right)=\mathfrak{K}\left(t-t^{*}\right), t^{*}>0$ (see, [42]). A special case of (15) is

$$
\frac{d \mathfrak{K}_{i}}{d t}=-\mathfrak{c}_{i} \mathfrak{K}_{i}(t),
$$

with $\mathfrak{K}_{i}(t=0)=\mathfrak{K}_{0}, \mathfrak{c}_{i}>0$ and the solution of $(16)$ is

$$
\mathfrak{K}_{i}(t)=\mathfrak{K}_{0} e^{-\mathfrak{c}_{i} t} .
$$

Performing the integration of (16) leads to

$$
\mathfrak{K}(t)-\mathfrak{K}_{0}=-\mathfrak{c}_{0} \mathfrak{D}_{t}^{-1} \mathfrak{K}(t),
$$

where ${ }_{0} \mathfrak{D}_{t}^{-1}$ is the particular case of Riemann-Liouville (R-L) integral operator and $\mathfrak{c}$ is a constant. The fractional form of (18) is (see [42])

$$
\mathfrak{K}(t)-\mathfrak{K}_{0}=-\mathfrak{c}^{\mu}{ }_{0} \mathfrak{D}_{t}^{-\mu} \mathfrak{K}(t),
$$

where $\mathfrak{D}_{t}^{-\mu}$ is given by

$$
{ }_{0} \mathfrak{D}_{t}^{-\mu} f(t)=\frac{1}{\Gamma(\mu)} \int_{0}^{t}(t-s)^{\mu-1} f(s) d s, \Re(\mu)>0 .
$$

Theorem 3.1. For $\varpi, \lambda, \gamma \in \mathbb{C}, \delta \neq 0,-1,-2, \cdots, d>0, \epsilon>0$ then the solution of

$$
\mathfrak{K}(t)-\mathfrak{K}_{0} \mathrm{E}^{\mathrm{E} ; \delta} \mathrm{E}^{\varpi, \lambda ; \gamma}(t)=-d^{\epsilon}{ }_{0} \mathfrak{D}_{t}^{-\epsilon} \mathfrak{K}(t)
$$

is given by

$$
\mathfrak{K}(t)=\mathfrak{K}_{0} \sum_{n=0}^{\infty} \frac{\left(\kappa_{1}\right)_{n} \ldots\left(\kappa_{p}\right)_{n}}{\left(\omega_{1}\right)_{n} \ldots\left(\omega_{q}\right)_{n}} \frac{(\gamma)_{n} n !}{(\delta)_{n} \Gamma(\varpi n+\lambda)} t^{n-1} E_{\epsilon, n}\left(-d^{\epsilon} t^{\epsilon}\right)
$$

Proof: The Sumudu transform (ST) of the R-L fractional operator is

$$
\mathcal{S}\left\{\mathfrak{o}_{t}^{\epsilon} g(t) ; u\right\}=u^{\epsilon} G(u)
$$

where $G(u)$ is defined in (14). Now, applying the ST on the both sides of (21) and using (7) and (23), we have

$$
\mathcal{S}\{\mathfrak{K}(t) ; u\}-\mathfrak{K}_{0} \mathcal{S}\left\{{ }_{p} \mathrm{E}_{q ; \delta}^{\varpi, \lambda ; \gamma}(t) ; u\right\}=\mathcal{S}\left\{-d^{\epsilon}{ }_{0} \mathfrak{D}_{t}^{-\epsilon} \mathfrak{K}(t) ; u\right\}, \quad(24)
$$


which gives

$$
\begin{aligned}
\mathfrak{K}^{*}(u) & =\mathfrak{K}_{0}\left(\int_{0}^{\infty} e^{-t} \sum_{n=0}^{\infty} \frac{\left(\kappa_{1}\right)_{n}\left(\kappa_{2}\right)_{n} \cdots\left(\kappa_{p}\right)_{n}}{\left(\omega_{1}\right)_{n}\left(\omega_{2}\right)_{n} \cdots\left(\omega_{q}\right)_{n}} \frac{(\gamma)_{n}(u t)^{n}}{(\delta)_{r} \Gamma(\varpi n+\lambda)} d t\right) \\
& -d^{\epsilon} u^{\epsilon} \mathfrak{K} *(u),
\end{aligned}
$$

which implies that

$$
\begin{aligned}
& \mathfrak{K}^{*}(u)\left[1+d^{\epsilon} u^{\epsilon}\right] \\
& =\mathfrak{K}_{0} \sum_{n=0}^{\infty} \frac{\left(\kappa_{1}\right)_{n}\left(\kappa_{2}\right)_{n} \cdots\left(\kappa_{p}\right)_{n}}{\left(\omega_{1}\right)_{n}\left(\omega_{2}\right)_{n} \cdots\left(\omega_{q}\right)_{n}} \frac{(\gamma)_{n}(u)^{n}}{(\delta)_{n} \Gamma(\varpi n+\lambda)} \\
& \int_{0}^{\infty} e^{-t} t^{n} d t .
\end{aligned}
$$

After some simple calculation, we get

$$
\begin{aligned}
\mathfrak{K}^{*}(u) & =\mathfrak{K}_{0} \sum_{n=0}^{\infty} \frac{\left(\kappa_{1}\right)_{n}\left(\kappa_{2}\right)_{n} \cdots\left(\kappa_{p}\right)_{n}}{\left(\omega_{1}\right)_{n}\left(\omega_{2}\right)_{n} \cdots\left(\omega_{q}\right)_{n}} \\
& \frac{(\gamma)_{n}}{(\delta)_{n} \Gamma(\varpi n+\lambda)\left(u^{n}\right) n !} \Gamma(n+1) \\
& \times\left\{\sum_{s=0}^{\infty}\left[(-d u)^{\epsilon}\right]^{s}\right\} .
\end{aligned}
$$

The inverse ST of (27) and using the formula $\mathcal{S}^{-1}\left\{u^{\epsilon} ; t\right\}=\frac{t^{\epsilon-1}}{\Gamma(\epsilon)}$, $\Re(\epsilon)>0$ gives

$$
\begin{aligned}
\mathfrak{K}(t) & =\mathfrak{K}_{0} \sum_{n=0}^{\infty} \frac{\left(\kappa_{1}\right)_{n}\left(\kappa_{2}\right)_{n} \cdots\left(\kappa_{p}\right)_{n}}{\left(\omega_{1}\right)_{n}\left(\omega_{2}\right)_{n} \cdots\left(\omega_{q}\right)_{n}} \frac{(\gamma)_{n}}{(\delta)_{n} \Gamma(\varpi n+\lambda)} \Gamma(n+1) \\
& \times \sum_{s=0}^{\infty}(-1)^{s} d^{\epsilon s} \frac{t^{\epsilon s+n-1}}{\Gamma(\epsilon s+n)} .
\end{aligned}
$$

In view of the Mittag-Leffler function definition, we arrived the needful result.

Theorem 3.2. For $\varpi, \lambda, \gamma \in \mathbb{C}, \delta \neq 0,-1,-2, \cdots, d>0, \epsilon>0$ then the equation

$$
\mathfrak{K}(t)-\mathfrak{K}_{0 p} \mathrm{E}_{q ; \delta}^{\varpi, \lambda ; \gamma}\left(d^{\epsilon} t^{\epsilon}\right)=-d^{\epsilon}{ }_{0} \mathfrak{D}_{t}^{-\epsilon} \mathfrak{K}(t)
$$

have the following solution

$$
\mathfrak{K}(t)=\mathfrak{K}_{0} \sum_{n=0}^{\infty} \frac{\left(\kappa_{1}\right)_{n} \ldots\left(\kappa_{p}\right)_{n}}{\left(\omega_{1}\right)_{n} \ldots\left(\omega_{q}\right)_{n}} \frac{(\gamma)_{n} \Gamma(\epsilon n+1)}{(\delta)_{n} \Gamma(\varpi n+\lambda)}(d)^{\epsilon n} t^{n-1} E_{\epsilon, n}\left(-d^{\epsilon} t^{\epsilon}\right)
$$

Proof: Applying the Sumudu transform on the both sides of (29)

$$
\mathcal{S}\{\mathfrak{K}(t) ; u\}-\mathfrak{K}_{0} \mathcal{S}\left\{{ }_{p} \mathrm{E}_{q ; \delta}^{\varpi, \lambda ; \gamma}\left(d^{\epsilon} t^{\epsilon}\right) ; u\right\}=\mathcal{S}\left\{-d^{\epsilon}{ }_{0} \mathfrak{D}_{t}^{-\epsilon} \mathfrak{K}(t) ; u\right\},
$$

and using (7) and (23), we get

$$
\mathfrak{K}^{*}(u)=\mathfrak{K}_{0}\left(\int_{0}^{\infty} e^{-t} \sum_{n=0}^{\infty} \frac{\left(\kappa_{1}\right)_{n}\left(\kappa_{2}\right)_{n} \cdots\left(\kappa_{p}\right)_{n}}{\left(\omega_{1}\right)_{n}\left(\omega_{2}\right)_{n} \cdots\left(\omega_{q}\right)_{n}} \frac{(\gamma)_{n}\left(u d^{\epsilon} t^{\epsilon}\right)^{n}}{(\delta)_{n} \Gamma(\varpi n+\lambda)} d t\right)
$$

$$
-d^{\epsilon} u^{\epsilon} \mathfrak{K}(u),
$$

which gives

$$
\begin{aligned}
\mathfrak{K}(u)[1+ & \left.d^{\epsilon} u^{\epsilon}\right] \\
& =\mathfrak{K}_{0} \sum_{n=0}^{\infty} \frac{\left(\kappa_{1}\right)_{n}\left(\kappa_{2}\right)_{n} \cdots\left(\kappa_{p}\right)_{n}}{\left(\omega_{1}\right)_{n}\left(\omega_{2}\right)_{n} \cdots\left(\omega_{q}\right)_{n}} \frac{(\gamma)_{n} u^{n} d^{\epsilon n}}{(\delta)_{n} \Gamma(\varpi n+\lambda)} \\
& \int_{0}^{\infty} e^{-t} t^{\epsilon n} d t,
\end{aligned}
$$

which can be simplified as

$$
\begin{aligned}
\mathfrak{K}(u) & =\mathfrak{K}_{0} \sum_{n=0}^{\infty} \frac{\left(\kappa_{1}\right)_{n}\left(\kappa_{2}\right)_{n} \cdots\left(\kappa_{p}\right)_{n}}{\left(\omega_{1}\right)_{n}\left(\omega_{2}\right)_{n} \cdots\left(\omega_{q}\right)_{n}} \frac{(\gamma)_{n} d^{\epsilon n}}{(\delta)_{n} \Gamma(\varpi n+\lambda)} \Gamma(\epsilon n+1) \\
& \times\left\{u^{n} d^{\epsilon n} \sum_{s=0}^{\infty}\left[(-d u)^{\epsilon}\right]^{s}\right\} .
\end{aligned}
$$

Taking the Sumudu inverse of (34) and using $\mathcal{S}^{-1}\left\{u^{\vartheta} ; t\right\}=\frac{t^{\vartheta-1}}{\Gamma(\vartheta)}$, we get

$$
\begin{aligned}
\mathfrak{K}(t) & =\mathfrak{K}_{0} \sum_{n=0}^{\infty} \frac{\left(\kappa_{1}\right)_{n}\left(\kappa_{2}\right)_{n} \cdots\left(\kappa_{p}\right)_{n}}{\left(\omega_{1}\right)_{n}\left(\omega_{2}\right)_{n} \cdots\left(\omega_{q}\right)_{n}} \frac{(\gamma)_{n} d^{\epsilon n} t^{n-1}}{(\delta)_{n} \Gamma(\varpi n+\lambda)} \Gamma(\epsilon n+1) \\
& \times \sum_{s=0}^{\infty}(-1)^{s} d^{\epsilon s} \frac{t^{\epsilon s}}{\Gamma(\epsilon s+1)} .
\end{aligned}
$$

In view of the definition of the Mittag-Leffler function, we get the required result.

Theorem 3.3. For $\varpi, \lambda, \gamma \in \mathbb{C}, \delta \neq 0,-1,-2, \cdots, d>0, \epsilon>0$ and $a \neq d$ then the equation

$$
\mathfrak{K}(t)-\mathfrak{K}_{0 p} \mathrm{E}_{q ; \delta}^{\varpi, \lambda ; \gamma}\left(d^{\epsilon} t^{\epsilon}\right)=-a^{\epsilon}{ }_{0} \mathfrak{D}_{t}^{-\epsilon} \mathfrak{K}(t)
$$

have the following solution

$$
\mathfrak{K}(t)=\mathfrak{K}_{0} \sum_{n=0}^{\infty} \frac{\left(\kappa_{1}\right)_{n} \ldots\left(\kappa_{p}\right)_{n}}{\left(\omega_{1}\right)_{n} \ldots\left(\omega_{q}\right)_{n}} \frac{(\gamma)_{n} \Gamma(\epsilon n+1)}{(\delta)_{n} \Gamma(\varpi n+\lambda)}(d)^{\epsilon n} t^{n-1} E_{\epsilon, n}\left(-a^{\epsilon} t^{\epsilon}\right)
$$

Proof: Applying the Sumudu transform on the both sides of (36)

$$
\mathcal{S}\{\mathfrak{K}(t) ; u\}-\mathfrak{K}_{0} \mathcal{S}\left\{{ }_{p} \mathrm{E}_{q ; \delta}^{\varpi, \lambda ; \gamma}\left(d^{\epsilon} t^{\epsilon}\right) ; u\right\}=\mathcal{S}\left\{-a^{\epsilon}{ }_{0} \mathfrak{D}_{t}^{-\epsilon} \mathfrak{K}(t) ; u\right\},
$$

and using (7) and (23), we get

$\mathfrak{K}^{*}(u)$

$$
\begin{aligned}
& =\mathfrak{K}_{0}\left(\int_{0}^{\infty} e^{-t} \sum_{n=0}^{\infty} \frac{\left(\kappa_{1}\right)_{n}\left(\kappa_{2}\right)_{n} \cdots\left(\kappa_{p}\right)_{n}}{\left(\omega_{1}\right)_{n}\left(\omega_{2}\right)_{n} \cdots\left(\omega_{q}\right)_{n}} \frac{(\gamma)_{n}\left(u d^{\epsilon} t^{\epsilon}\right)^{n}}{(\delta)_{n} \Gamma(\varpi n+\lambda)} d t\right) \\
& -a^{\epsilon} u^{\epsilon} \mathfrak{K}(u),
\end{aligned}
$$

which gives

$\mathfrak{K}(\rho)\left[1+a^{\epsilon} u^{\epsilon}\right]$ 


$$
\begin{aligned}
& =\mathfrak{K}_{0} \sum_{n=0}^{\infty} \frac{\left(\kappa_{1}\right)_{n}\left(\kappa_{2}\right)_{n} \cdots\left(\kappa_{p}\right)_{n}}{\left(\omega_{1}\right)_{n}\left(\omega_{2}\right)_{n} \cdots\left(\omega_{q}\right)_{n}} \frac{(\gamma)_{n} u^{n} d^{\epsilon n}}{(\delta)_{n} \Gamma(\varpi n+\lambda)} \\
& \int_{0}^{\infty} e^{-t} t^{\epsilon n} d t
\end{aligned}
$$

which can be simplified as

$$
\begin{aligned}
\mathfrak{K}(u) & =\mathfrak{K}_{0} \sum_{n=0}^{\infty} \frac{\left(\kappa_{1}\right)_{n}\left(\kappa_{2}\right)_{n} \cdots\left(\kappa_{p}\right)_{n}}{\left(\omega_{1}\right)_{n}\left(\omega_{2}\right)_{n} \cdots\left(\omega_{q}\right)_{n}} \frac{(\gamma)_{n} d^{\epsilon n}}{(\delta)_{n} \Gamma(\varpi n+\lambda)} \Gamma(\epsilon n+1) \\
& \times\left\{u^{n} d^{\epsilon n} \sum_{s=0}^{\infty}\left[(-a u)^{\epsilon}\right]^{s}\right\} .
\end{aligned}
$$

Taking the Sumudu inverse of (41) and using $\mathcal{S}^{-1}\left\{u^{\vartheta} ; t\right\}=\frac{t^{\vartheta-1}}{\Gamma(\vartheta)}$, we get

$$
\begin{aligned}
\mathfrak{K}(t) & =\mathfrak{K}_{0} \sum_{n=0}^{\infty} \frac{\left(\kappa_{1}\right)_{n}\left(\kappa_{2}\right)_{n} \cdots\left(\kappa_{p}\right)_{n}}{\left(\omega_{1}\right)_{n}\left(\omega_{2}\right)_{n} \cdots\left(\omega_{q}\right)_{n}} \frac{(\gamma)_{n} d^{\epsilon n} t^{n-1}}{(\delta)_{n} \Gamma(\varpi n+\lambda)} \Gamma(\epsilon n+1) \\
& \times \sum_{s=0}^{\infty}(-1)^{s} a^{\epsilon s} \frac{t^{\epsilon s}}{\Gamma(\epsilon s+1)} .
\end{aligned}
$$

In view of the definition of the Mittag-Leffler function, we get the required result.

If we take $\delta=1$ in Theorem 3.1, we get the generalized FKE involving $K$ - function as follows:

Corollary 3.1. For $\varpi, \lambda, \gamma \in \mathbb{C}, \delta \neq 0,-1,-2, \cdots, d>0$, $\epsilon>0$ then

$$
\mathfrak{K}(t)-\mathfrak{K}_{0} p^{E_{q ; 1}^{\varpi, \lambda ; \gamma}}(t)=-d^{\epsilon}{ }_{0} \mathfrak{D}_{t}^{-\epsilon} \mathfrak{K}(t)
$$

is given by

$$
\mathfrak{K}(t)=\mathfrak{K}_{0} \sum_{n=0}^{\infty} \frac{\left(\kappa_{1}\right)_{n} \ldots\left(\kappa_{p}\right)_{n}}{\left(\omega_{1}\right)_{n} \ldots\left(\omega_{q}\right)_{n}} \frac{(\gamma)_{n}}{\Gamma(\varpi n+\lambda)} t^{n-1} E_{\epsilon, n}\left(-d^{\epsilon} t^{\epsilon}\right)
$$

If we take $\delta=1, p=q=0$ in Theorem 3.1, we have the generalized FKE involving the Prabhakar function:

Corollary 3.2. For $\varpi, \lambda, \gamma \in \mathbb{C}, \delta \neq 0,-1,-2, \cdots, d>0$, $\epsilon>0$ then

$$
\mathfrak{K}(t)-\mathfrak{K}_{0}{ }_{0} \mathrm{E}_{0 ; 1}^{\varpi, \lambda ; \gamma}(t)=-d^{\epsilon}{ }_{0} \mathfrak{D}_{t}^{-\epsilon} \mathfrak{K}(t)
$$

is given by

$$
\mathfrak{K}(t)=\mathfrak{K}_{0} \sum_{n=0}^{\infty} \frac{(\gamma)_{n}}{\Gamma(\varpi n+\lambda)} t^{n-1} E_{\epsilon, n}\left(-d^{\epsilon} t^{\epsilon}\right)
$$

If we choose $\delta=1, p=q=0$ and $\gamma=1$ in Theorem 3.1, then the generalized FKE involving the Wiman function:

Corollary 3.3. For $\varpi, \lambda \in \mathbb{C}, \delta \neq 0,-1,-2, \cdots, d>0$, $\epsilon>0$ then

$$
\mathfrak{K}(t)-\mathfrak{K}_{0}{ }_{0} \mathrm{E}_{0 ; 1}^{\varpi, \lambda ; 1}(t)=-d^{\epsilon}{ }_{0} \mathfrak{D}_{t}^{-\epsilon} \mathfrak{K}(t)
$$

is given by

$$
\mathfrak{K}(t)=\mathfrak{K}_{0} \sum_{n=0}^{\infty} \frac{1}{\Gamma(\varpi n+\lambda)} t^{n-1} E_{\epsilon, n}\left(-d^{\epsilon} t^{\epsilon}\right)
$$

If we choose $\delta=1, p=q=0$ and $\gamma=1$ in Theorem 3.1, then we get the generalized FKE involving the Mittag-Leffler function:

Corollary 3.4. For $\varpi \in \mathbb{C}, \delta \neq 0,-1,-2, \cdots, d>0, \epsilon>0$ then

$$
\mathfrak{K}(t)-\mathfrak{K}_{0}{ }_{0} \mathrm{E}_{0 ; 1}^{\sigma, 1 ; 1}(t)=-d^{\epsilon}{ }_{0} \mathfrak{D}_{t}^{-\epsilon} \mathfrak{K}(t)
$$

is given by

$$
\mathfrak{K}(t)=\mathfrak{K}_{0} \sum_{n=0}^{\infty} \frac{1}{\Gamma(\varpi n+1)} t^{n-1} E_{\epsilon, n}\left(-d^{\epsilon} t^{\epsilon}\right)
$$

Remark 3.1. By choosing the suitable parameters in Theorems 3.2 and 3.3, one can derive the generalized FKEs of GMLTF as similar as above corollaries.

\section{CONCLUSION}

The generalized fractional integrations of the generalized MittagLeffler type function is studied in this paper. The obtained results are expressed in terms of the generalized Wright hypergeometric function and generalized hypergeometric functions. To show the potential application of GMLTF, the solutions of fractional kinetic equations are derived with the help of Sumudu transform. The results obtained in this study have significant importance as the solution of the equations are general and can derive many new and known solutions of FKEs involving various type of special functions.

\section{DATA AVAILABILITY STATEMENT}

All datasets generated for this study are included in the article.

\section{AUTHOR CONTRIBUTIONS}

The author confirms being the sole contributor of this work and has approved it for publication.

\section{REFERENCES}

1. Srivastava HM, Choi J. Zeta and q-Zeta Functions and Associated Series and Integrals. Amsterdam; London; New York, NY: Elsevier Science Publishers (2012).

2. Rainville ED. Special Functions. New York, NY: Macmillan (1960).

3. Fox C. The asymptotic expansion of generalized hypergeometric functions. Proc Lond Math Soc. (1928) 27:389-400.

4. Kilbas AA, Saigo M, Trujillo JJ. On the generalized Wright function. Fract Calc Appl Anal. (2002) 5:437-60. 
5. Wright EM. The asymptotic expansion of integral functions defined by Taylor series. Philos Trans Roy Soc Lond A. (1940) 238:423-51.

6. Wright EM. The asymptotic expansion of the generalized hypergeometric function. Proc Lond Math Soc. (1940) 46:389-408.

7. Mittag-Leffler GM. Sur la representation analytiqie d'une fonction monogene cinquieme note. Acta Math. (1905) 29:101-81.

8. Wiman A. Uber den fundamental satz in der theorie der funktionen $E_{\alpha}(z)$. Acta Math. (1905) 29:191-201.

9. Rahman G, Baleanu D, Al Qurashi M, Purohit SD, Mubeen S, Muhammad A. The extended Mittag-Leffler function via fractional calculus. J Nonlinear Sci Appl. (2017) 10:4244-53. doi: 10.22436/jnsa.010.08.19

10. Nisar KS, Rahman G, Baleanu D, Mubeen S, Arshad M. The $(k, s)$-fractional calculus of k-Mittag-Leffler function. Adv Differ Equat. (2017) 2017:118. doi: 10.1186/s13662-017-1176-4

11. Kumar S, Kumar A, Momani S, Aldhaifallah M, Nisar KS. Numerical solutions of nonlinear fractional model arising in the appearance of the stripe patterns in two-dimensional systems. Adv Differ Equat. (2019) 2019:413. doi: 10.1186/s13662-019-2334-7

12. Singh J, Kumar D, Swroop R, Kumar S. An efficient computational approach for time-fractional Rosenau-Hyman equation. Neural Comput Appl. (2018) 30:3063-70. doi: 10.1007/s00521-017-2909-8

13. Bhatter S, Mathur A, Kumar D, Nisar KS, Singh J. Fractional modified Kawahara equation with Mittag-Leffler law. Chaos Solit Fract. (2019) 2019:109508. doi: 10.1016/j.chaos.2019.109508

14. Nisar KS. Fractional integrations of a generalized Mittag-Leffler type function and its application. Mathematics. (2019) 7:1230. doi: 10.3390/math7121230

15. Sharma K. Application of fractional calculus operators to related Areas. Gen Math Notes. (2011) 7:33-40. doi: 10.1142/9789814340250_0005

16. Prabhakar TR. A singular integral equation with a generalized Mittag-Leffler function in the Kernel. Yokohama Math J. (1971) 19:7-15.

17. Srivastava HM, Tomovski Z. Fractional calculus with an integral operator containing a generalized Mittag-Leffler function in the kernel. Appl Math Comput. (2009) 211:198-210. doi: 10.1016/j.amc.2009.01.055

18. Odibat Z, Kumar S. A robust computational algorithm of homotopy asymptotic method for solving systems of fractional differential equations. $J$ Comput Nonlinear Dyn. (2019) 14:081004. doi: 10.1115/1.4043617

19. Singh J, Kumar D, Baleanu D. New aspects of fractional Biswas-Milovic model with Mittag-Leffler law. Math Model Nat Phenom. (2019) 14:303. doi: $10.1051 / \mathrm{mmnp} / 2018068$

20. Yang XJ. New general fractional-order rheological models with kernels of Mittag-Leffler functions. Rom Rep Phys. (2017) 69:118.

21. Yang XJ, Gao F, Tenreiro Machado JA, Baleanu D. A new fractional derivative involving the normalized sinc function without singular kernel. Eur Phys J Spec Top. (2017) 226:3567-75. doi: 10.1140/epjst/e2018-00020-2

22. Gao W, Ghanbari B, Baskonus HM. New numerical simulations for some real world problems with Atangana-Baleanu fractional derivative. Chaos Solit Fract. (2019) 128:34-43. doi: 10.1016/j.chaos.2019.07.037

23. Araci S, Rahman G, Ghaffar A, Azeema, Nisar KS. Fractional calculus of extended Mittag-Leffler function and its applications to statistical distribution. Mathematics. (2019) 7:248. doi: 10.3390/math7030248

24. Andrić M, Farid G, Pećarić J. A further extension of Mittag-Leffler function. Fract Calc Appl Anal. (2018) 21:1377-95. doi: 10.1515/fca-2018-0072

25. Rahman G, Nisar KS, Choi J, Mubeen S, Arshad M. Formulas for Saigo fractional integral operators with $2 \mathrm{~F} 1$ generalized $\mathrm{k}$-Struve functions. Far East J Math Sci. (2017) 102:55-66. doi: 10.17654/MS102010055

26. Kumar D, Singh J, Baleanu D. A new analysis of the Fornberg-Whitham equation pertaining to a fractional derivative with Mittag-Leffler-type kernel. Eur Phys J Plus. (2018) 133:70. doi: 10.1140/epjp/i2018-11934-y

27. Choi J, Mubeen S, Nisar KS, Choi J. Certain extended special functions and fractional integral and derivative operators via an extended beta function. Nonlinear Funct Anal Appl. (2019) 24:1-13. Available online at: http://nfaa. kyungnam.ac.kr/journal-nfaa
28. Kiryakova V. All the special functions are fractional differintegrals of elementary functions. J Phys A. (1977) 30:5085-103.

29. Miller KS, Ross B. An Introduction to the Fractional Calculus and Fractional Differential Equations. New York, NY: Wiley (1993).

30. Srivastava HM, Lin SD, Wang PY. Some fractional-calculus results for the Hfunction associated with a class of Feynman integrals. Russ J Math Phys. (2006) 13:94-100. doi: 10.1134/S1061920806010092

31. Marichev OI. Volterra equation of Mellin convolution type with a Horn function in the kernel. Izvest Akad Nauk BSSR Ser Fiziko Mat Nauk. (1974) 1:128-9.

32. Saigo M, Maeda N. Editors. More generalization of fractional calculus. Transform methods and special functions. In: Proceedings of the 2 nd International Workshop, Varna (1996).

33. Baleanu D, Kumar D, Purohit SD. Generalized fractional integrals of product of two $\mathrm{H}$-functions and a general class of polynomials. Int J Comput Math. (2016) 93:1320-9. doi: 10.1080/00207160.2015.1045886

34. Mondal SR, Nisar KS. Marichev-Saigo-Maeda fractional integration operators involving generalized Bessel functions. Math Probl Eng. (2014) 2014:11. doi: 10.1155/2014/274093

35. Purohit SD, Suthar DL, Kalla SL. Marichev-Saigo-Maeda fractional integration operators of the Bessel function. Le Mat. (2012) 67:21-32. doi: $10.4418 / 2012.67 .1 .2$

36. Kataria KK, Vellaisamy P. The generalized $k$-Wright function and MarichevSaigo-Maeda fractional operators. J Anal. (2015) 23:75-87.

37. Watugala GK. Sumudu transform:a new integral transform to solve differential equations and control engineering problems. Int J Math Edu Sci Tech. (1993) 24:35-43.

38. Watugala GK. The Sumudu transform for functions of two variables. Math Eng Ind. (2002) 8:293-302.

39. Asiru MA. Sumudu transform and the solution of integral equation of convolution type. Int J Math Educ Sci Technol. (2001) 32:906-10. doi: 10.1080/002073901317147870

40. Belgacem FBM, Karaballi AA, Kalla SL. Analytical investigations of the Sumudu transform and applications to integral production equations. J Math Probl Eng. (2003) 3:103-18. doi: 10.1155/S1024123X03207018

41. Bulut $\mathrm{H}$, Baskonus HM, Belgacem FB. The analytical solution of some fractional ordinary differential equations by the Sumudu transform method. Abstr Appl Anal. (2013) 2013:6. doi: 10.1155/2013/203875

42. Haubold HJ, Mathai AM. The fractional kinetic equation and thermonuclear functions. Astrophys Space Sci. (2000) 327:53-63. doi: 10.1023/A:1002695807970

43. Saxena RK, Mathai AM, Haubold HJ. On fractional kinetic equations. Astrophys Space Sci. (2002) 282:281-7. doi: 10.1023/A:1021175108964

44. Saxena RK, Mathai AM, Haubold HJ. On generalized fractional kinetic equations. Phys A. (2004) 344:657-64. doi: 10.1016/j.physa.2004. 06.048

45. Nisar KS, Purohit SD, Mondal SR. Generalized fractional kinetic equations involving generalized Struve function of the first kind J King Saud Univ Sci. (2016) 28:167-71. doi: 10.1016/j.jksus.201 5.08 .005

Conflict of Interest: The author declares that the research was conducted in the absence of any commercial or financial relationships that could be construed as a potential conflict of interest.

Copyright $(2020$ Nisar. This is an open-access article distributed under the terms of the Creative Commons Attribution License (CC BY). The use, distribution or reproduction in other forums is permitted, provided the original author(s) and the copyright owner(s) are credited and that the original publication in this journal is cited, in accordance with accepted academic practice. No use, distribution or reproduction is permitted which does not comply with these terms. 